\title{
ESCAPE-CAPTURE BIGEMINY
}

\author{
MECHANISMS IN S-A BLOCK, A-V BLOCK, AND REVERSED \\ RECIPROCAL RHYTHM \\ BY
}

\section{SCHAMROTH AND A. DUBB}

From the Baragwanath Hospital and University of the Witwatersrand, Johannesburg, S. Africa

Received August 10, 1964

The term escape-capture bigeminy was coined by Bradley and Marriott (1958) in preference to "pseudo-reciprocal rhythm". This arrhythmia simulates reciprocal rhythm in having a P wave "sandwiched" between two QRS complexes. It differs from true reciprocal rhythm, however, in that the $\mathbf{P}$ wave is normal and does not result from retrograde activation. The purpose of this paper is to present three different mechanisms that can produce this arrhythmia.

\section{Mechanism I: EsCAPe-CAPTURe Bigeminy INITIATED By S-A BLock}

Case 1. The electrocardiograms are those of a 45-year-old man with congestive cardiomyopathy on full digitalis dosage. The electrocardiogram in Fig. 1 shows P-P intervals of approximately 285 hundredths of a second. (All subsequent time intervals are expressed in hundredths of a second, i.e. 220=220 hundredths of a second.) A ventricular escape beat follows each conducted sinus impulse at an escape interval of 220 (range: 195-230). The sinus impulses occur just after the refractory period of the ventricular escape beats (shaded area) and are able to activate the ventricles once again. Sinus impulses that occur relatively late after the escape beats (the first $\mathbf{P}$ wave in the upper strip and the second $\mathbf{P}$ wave in the lower strip of Fig. 1) are conducted normally. Sinus impulses that occur relatively early after the escape beats are conducted with phasic aberrant ventricular conduction (Schamroth and Chesler, 1963). The escape beat followed by a conducted (capture) beat results in escape-capture bigeminy.

The P-P interval of 285 is too long for a sinus bradycardia and suggests the presence of second degree S-A block. This was confirmed two days later (Fig. 2) when the P-P interval was found to be 190 . This interval is two-thirds of the previous P-P interval of 285 thus indicating that (a) the P-P interval of 285 represents a 3:1 S-A block $(3 \times 95)$, and (b) the P-P interval of 190 represents a 2:1 S-A block $(2 \times 95)$. As the P-P interval of 190 is less than the ventricular escape interval of 220 , the escape rhythm is abolished.

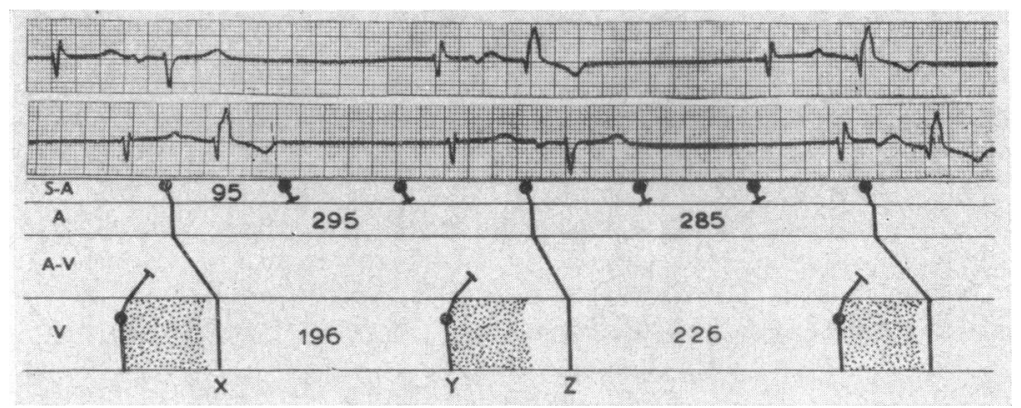

FIG. 1.-Case 1. Electrocardiogram (continuous strip of lead V1) showing escape-capture bigeminy resulting from 3:1 S-A block (see text). The diagram refers to the lower strip only. 


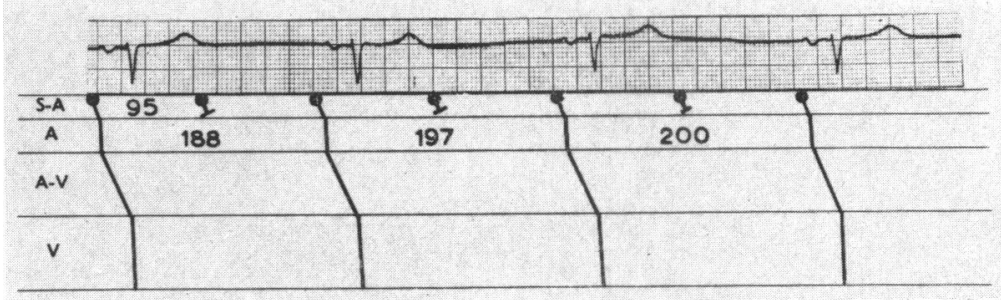

FIG. 2.-Case 1. Electrocardiogram (lead V1) showing 2:1 S-A block (see text).

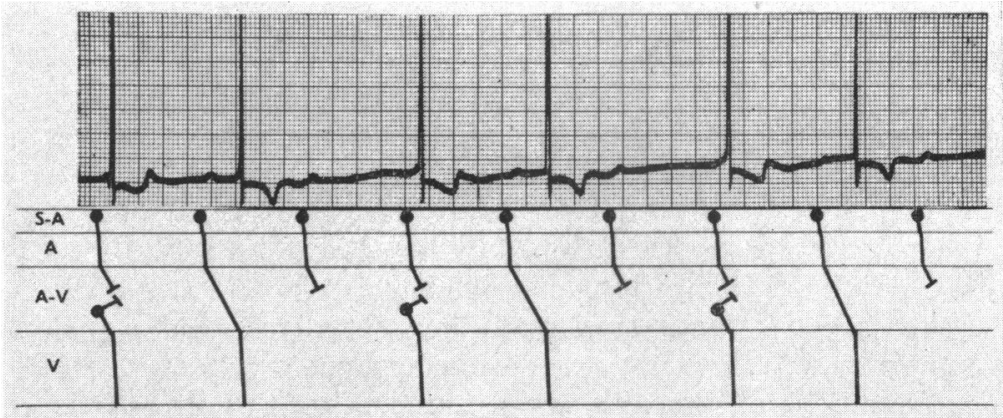

FIG. 3.-Case 2. Electrocardiogram (lead V5) showing escape-capture bigeminy resulting from 3:2 A-V block (see text).

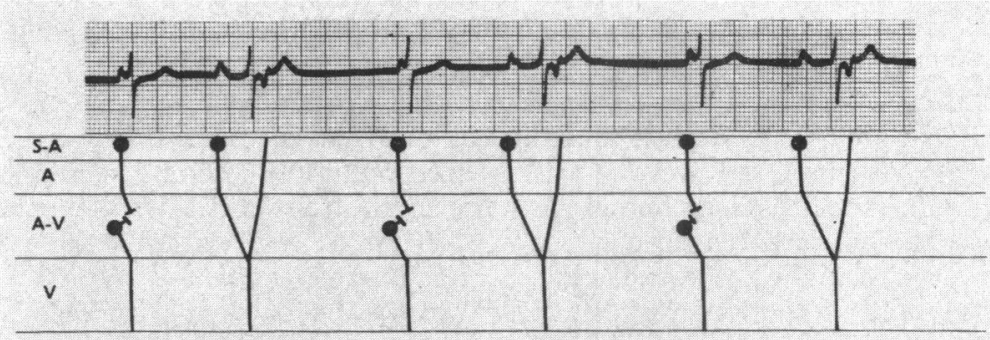

FIG. 4.-Case 3. Electrocardiogram (standard lead II) showing escape-capture bigeminy resulting from reversed reciprocal rhythm (see text). (From Schamroth: American Heart Journal, 59, 506, 1960, published by The C.U. Mosby Company, St Louis, Missouri, U.S.A.)

\section{Mechanism II: EsCAPE-CAPTURe Bigeminy INITIATED by A-V Block}

Case 2 (Fig. 3). The electrocardiogram was recorded from a patient with hypertensive heart failure on full digitalis dosage. The rhythm begins with a dissociated beat (the $\mathbf{P}$ wave is seen just before and superimposed on the first QRS complex). The next sinus impulse is conducted with a prolonged P-R interval. The third sinus impulse is blocked, and this permits the A-V node to escape, resulting in another dissociated beat. The basic rhythm is thus a 3:2 A-V block, the blocked beat permitting the A-V node to escape. The escape beat followed by a conducted (capture) beat results in escape-capture bigeminy.

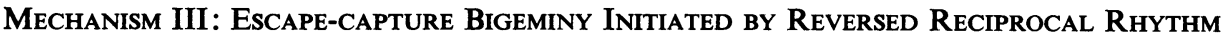

Case 3 (Fig. 4). The electrocardiogram is that of a 20-year-old man who complained of palpitations. There was no evidence of heart disease, nor had he taken any cardiac drugs. The bigeminal rhythm in this case is due to a very rare mechanism-reversed reciprocal rhythm. This case has been described in detail elsewhere (Schamroth, 1960). 
The electrocardiogram (standard lead II) begins with a dissociated beat-the $\mathbf{P}$ wave appears just before the first QRS complex. The following sinus impulse is conducted with first degree A-V block. This impulse, however, returns retrogradely through an accessory pathway to activate the atria once again. This results in an inverted ("retrograde") $P$ wave. The retrograde impulse discharges the S-A node prematurely and thus postpones the next sinus impulse. The next intersinus interval is longer than the preceding one, due to post-extrasystolic depression (Pick, Langendorf, and Katz, 1951): premature discharge of a pacemaker results in its transient depression. In this case, the S-A node is discharged extremely prematurely by the retrograde impulse, resulting in its transient depression. This postponement of the next sinus beat permits the A-V node to escape. The sequences are repeated, resulting in escape-capture bigeminy.

\section{Discussion}

Escape-capture bigeminy can only occur when there is a marked discrepancy between the escape interval and the effective intersinus interval. The effective intersinus interval is defined as the interval between sinus impulses at the level of a lower pacemaker. For escape-capture bigeminy to occur, the discrepancy between the two pacemakers must be such that the effective intersinus interval exceeds the sum of the escape interval and the refractory period following the escape beat. This requires an intermittent block of sinus impulses at either the sinus or A-V nodal levels.

Three mechanisms for this phenomenon have been illustrated.

In Case 1 the escape-capture bigeminy is due to S-A block which results in a long intersinus interval at the lower pacemaker (ventricular) level. The intersinus interval at ventricular level ( $\mathrm{X}-\mathrm{Z}$ in Fig. 1) exceeds the sum of the escape interval (X-Y) and the subsequent ventricular refractory period (shaded area). If the intersinus interval were less than this, some of the sinus impulses would occur during the refractory period of the escaping pacemaker, resulting in a different form of A-V dissociation. This form of escape-capture bigeminy is extremely rare. A similar case was described by Bradley and Marriott (1958) who could find but one other published example (Padilla and Cossio, 1930).

In the second case, escape-capture bigeminy is due to A-V block; two of every three sinus impulses are blocked in the A-V node, resulting in an effective $3: 2$ block at the level of the escaping (lower nodal) pacemaker. This form of escape-capture bigeminy is common; we have seen 8 examples in the past year.

In the third case, escape-capture bigeminy is due to reversed reciprocal rhythm-the effective intersinus interval is increased by the reversed reciprocal impulse which discharges the S-A node prematurely and thus delays the onset of the next sinus impulse. This mechanism is extremely rare, this case being the only published example.

\section{SUMMARY}

Three mechanisms of escape-capture bigeminy are described and illustrated. This arrhythmia may result from S-A block, A-V block, and reversed reciprocal rhythm.

We wish to thank Dr. W. Cooper, Coronation Hospital, for permission to publish Case 1, and the Medical Superintendent, Baragwanath Hospital, for permission to publish this paper.

We are grateful to the Photographic Unit, Department of Medicine, University of the Witwatersrand, for reproducing the electrocardiograms.

\section{REFERENCES}

Bradley, S. M., and Marriott, H. J. L. (1958). Escape-capture bigeminy. Report of a case of A-V dissociation initiated by 2:1 S-A block with resulting bigeminal rhythm. Amer. J. Cardiol., 1, 640.

Padilla, T., and Cossio, P. (1930). Disociación auriculo-ventricular por interferencia, con captura ventricular. La parasistolia más evidente. Sem. méd. (B. Aires), 37(1), 1412.

Pick, A., Langendorf, R., and Katz, L. N. (1951). Depression of cardiac pacemakers by premature impulses. Amer. Heart J., 41, 49.

Schamroth, L. (1960). Reversed reciprocating paroxysmal tachycardia and its relationship to the Wolff-ParkinsonWhite syndrome. Amer. Heart J., 59, 506.

—, and Chesler, E. (1963). Phasic aberrant ventricular conduction. Brit. Heart J., 25, 219. 